\title{
The Effects of Eccentric Exercise on Muscle Damage and Blood Redox Status in Men and Women
}

\author{
Michalis G. Nikolaidis \\ Department of Physical Education and Sports Sciences at Serres, Aristotle University of Thessaloniki, \\ Agios Ioannis, 62110 Serres, Greece; nikolaidis@auth.gr; Tel.: +30-23-1099-1086; Fax: +30-23-2106-4806
}

Received: 8 June 2017; Accepted: 16 June 2017; Published: 19 June 2017

\begin{abstract}
The purpose of the present investigation was to study the possible differences between men and women in muscle damage indices and oxidative stress biomarkers in response to eccentric exercise. Ten males and ten females performed a bout of eccentric exercise using an isokinetic dynamometer. Muscle damage indices (i.e., isometric torque, range of movement, delayed onset muscle soreness, and creatine kinase (CK)) and oxidative stress biomarkers (i.e., protein carbonyls and glutathione) were measured before and $48 \mathrm{~h}$ after eccentric exercise. No significant main effect of group or time-group interaction was found for muscle damage indices and oxidative stress biomarkers. However, the main effect of time was significant for all measured parameters. The findings of the present investigation indicate that eccentric exercise caused muscle damage and oxidative stress (i.e., protein and glutathione oxidation) in both males and females with no significant differences between the two groups. It is concluded that there are no differences between males and females regarding muscle damage and oxidative stress after eccentric exercise.
\end{abstract}

Keywords: delayed onset muscle soreness; isokinetic dynamometry; glutathione; protein carbonyls; range of motion

\section{Introduction}

Resistance exercise includes lengthening (eccentric) actions, which occur when the muscle unsuccessfully resists elongation, acting as a brake. However, lengthening the muscle during contraction may lead to muscle damage [1,2] and is characterized by sustained loss of range of motion $[3,4]$ as well as the development of delayed onset muscle soreness (DOMS) [2]. These changes typically peak at $1-3$ days after unaccustomed exercise and subside $4-7$ days after exercise $[5,6]$.

It is known that intense exercise can produce reactive oxygen species (ROS) that may cause oxidative stress especially when antioxidant defense capacity is not efficient to restrict their production [7]. Despite the many human studies investigating the effects of acute concentric non-muscle-damaging exercise on blood redox status (reviewed in [7]), our group focused on the effects of acute eccentric muscle-damaging exercise on blood redox status [3,8-11]. The main finding of these studies was that eccentric exercise provoked large and uniform alterations in several oxidative stress indices and antioxidant molecules, indicating increased oxidative stress, probably arising from increased free radical production in the damaged skeletal muscle fibers.

A few studies in the literature have compared males and females in response to unaccustomed muscle damaging eccentric exercise [12-14]. In the aforementioned studies, unaccustomed eccentric exercise induced parallel responses in some muscle damage indices (i.e., development and intensity of delayed onset muscle soreness and range of motion) but not in others (i.e., range of motion, torque recovery, and creatine kinase (CK)) between men and women. However, despite the non-significant differences between men and women in the pattern of response after eccentric exercise, the women showed more pronounced changes in range of motion [13], lower CK concentration in blood [12], 
while strength recovery was more rapid in women compared to men [14]. It is of interest that, despite the wealth of information about the effects of acute eccentric exercise on oxidative stress, much less is known about the possible differences between men and women, particularly when the effects of eccentric exercise are investigated in relation to muscle damage and oxidative stress in the same study. Based on the above, the purpose of the present investigation was to study the contrast between men and women in muscle damage indices and oxidative stress biomarkers after eccentric exercise. We used a global marker of systemic protein oxidation (i.e., protein carbonyls in plasma) and the most important non-enzymatic antioxidant molecule (reduced glutathione in erythrocytes).

\section{Materials and Methods}

\subsection{Participants}

Subjects were recruited after advertising the study in the local media. Ten males (age $22.4 \pm 3.1$ years; weight $74.8 \pm 7.2 \mathrm{~kg}$; height $177 \pm 5 \mathrm{~cm}$; BMI $23.9 \pm 2.2$; body fat $13.6 \pm 4.0 \%$ ) and 10 females (age $22.1 \pm 3.5$ years; weight $64.1 \pm 8.5 \mathrm{~kg}$; height $169 \pm 7 \mathrm{~cm}$; BMI $22.3 \pm 2.1$; body fat $22.4 \pm 2.1 \%$ ) participated in the study. Over the six months prior to their participation in the study, subjects had no experience of scheduled training that included intense eccentric muscle actions. Moreover, they were asked not to participate in any kind of strenuous exercise 3 days before and during the data collection. Subjects were also instructed to avoid any anti-inflammatory drugs and smoking. All female volunteers were eumenorrhoeic (reporting their menstrual cycle as lasting 24-30 days), while the eccentric exercise session was performed during the luteal phase of their menstrual cycle. The muscle damaging exercise trial fell within the luteal phase of the menstrual cycle ( 2 up to 6 days after ovulation), in an attempt to avoid any variance in estrogen levels among the women that participated in the study. The luteal phase of the cycle was chosen because it is relatively constant in all women, with a duration of 14 days [15]. Use of contraceptives was an additional exclusion criterion. This was because oral contraceptives have been found to affect the degree of muscle damage after eccentric exercise [16]. All subjects read and signed an informed consent form in which the possible risks and discomforts as well as the benefits of their participation in the study was described in detail. The study was conducted in accordance with the Declaration of Helsinki and approval was received from the Research Ethics Committee of the European University Cyprus (016/12-05-2013).

\subsection{Research Design}

The assessments of body mass (measured with accuracy of $0.5 \mathrm{~kg}$; Beam Balance 710, Seca, Birmingham, UK), standing body height (Stadiometer 208, Seca, Birmingham, UK), and percent body fat (Siri skinfold equation from seven measures using a Harpenden caliper (John Bull, West Sussex, UK)) were performed in accordance to published guidelines (American College of Sports Medicine, 2000) between 8:00 and 9:00 a.m. All participants using the knee extensors of the dominant leg undertook an isokinetic eccentric exercise protocol. Pain-free range of movement (ROM) and delayed onset muscle soreness (DOMS) during squat movement was evaluated before and $48 \mathrm{~h}$ after exercise. During the familiarization session, which was conducted the week before the experimental procedure, the subjects performed 8-10 eccentric muscle actions on the isokinetic dynamometer. The intensity of the 8-10 repetitions were of very low intensity insufficient to induce muscle damage.

\subsection{Isokinetic Exercise Protocol}

The isokinetic dynamometer (Cybex Norm, Ronkonkoma, NY, USA) was calibrated every week according to instructions provided by the manufacturer. The position of the subjects on the dynamometer (i.e., seated $\left(120^{\circ}\right.$ hip angle) with the lateral femoral condyle aligned with the rotation axis of the dynamometer while the ankle cuff of the dynamometer was attached proximal to the lateral malleolus) was recorded for the follow-up measurements. The functional range of motion of each participant was set between full extension 0 and $120^{\circ}$ of knee flexion to prevent hyperextension and 
hyperflexion, while gravitational corrections were performed in order to avoid the effect of limb weight on torque measurements. The dynamometer was providing full feedback information regarding the intensity and duration of every eccentric exercise session. A standard warm-up (i.e., 8 min cycling on a Monark cycle ergometer (Monark Excise AB, Vansbro, Sweden) at $70 \mathrm{rpm}$ and $50 \mathrm{~W}$ ) was performed before each assessment, while the eccentric contractions of the knee extensors were performed at an angular velocity of $60^{\circ} / \mathrm{s}$ (knee range, $\left(0^{\circ}\right.$ to and $\left.90^{\circ}\right)$ ). During the exercise session, subjects had to accomplish with their dominant leg five sets of 15 eccentric maximal voluntary contractions in the seated position as described previously [2]. A 2 min rest interval was incorporated between sets.

\subsection{Muscle Damage Indices}

The isokinetic dynamometer was used for the measurement of isometric knee extensor peak torque at $90^{\circ}$ knee flexion. The average of the 3 best maximal voluntary contractions with the preferred leg was recorded. To ensure that the subjects provided their maximal effort, the measurements were repeated if the difference between the lower and the higher torque values exceeded $10 \%$. There was a 2 min rest between isometric efforts. During the assessment of pain free ROM, which was performed on the same isokinetic dynamometer, the investigator moved the limb from full extension $\left(0^{\circ}\right)$ to the position were the participant started to feel pain. The movement of the limb was conducted with very slow angular velocity, and there was no pain for any of the subjects at the position of full extension $\left(0^{\circ}\right)$. The assessment of DOMS was performed using a squat movement $\left(90^{\circ}\right.$ knee flexion) during which volunteers subjectively evaluated their soreness. Perceived soreness was rated on a scale ranging from 1 (normal) to 10 (very sore) as described previously [10].

\subsection{Blood Collection}

Before and $48 \mathrm{~h}$ after the exercise protocol, blood samples were drawn from a forearm vein. The blood was collected into tubes containing EDTA. The blood was centrifuged immediately at $1370 \times g$ for $10 \mathrm{~min}$ at $4{ }^{\circ} \mathrm{C}$, and the plasma was collected. The packed erythrocytes were lysed with $1: 1(\mathrm{v} / \mathrm{v})$ distilled water, inverted vigorously, and centrifuged at $4000 \times \mathrm{g}$ for $15 \mathrm{~min}$ at $4{ }^{\circ} \mathrm{C}$.

\subsection{Assays}

Plasma protein carbonyls and erythrocyte glutathione (GSH) were determined spectrophotometrically [17]. Specifically, protein carbonyls were measured by adding $50 \mu \mathrm{L}$ of $20 \%$ TCA to $50 \mu \mathrm{L}$ of plasma or erythrocyte lysate (diluted 1:10). The mixture was placed in an ice tank for $15 \mathrm{~min}$ and centrifuged at $15,000 \times \mathrm{g}$ for $5 \mathrm{~min}$ at $4{ }^{\circ} \mathrm{C}$. The supernatant was removed, and $500 \mu \mathrm{L}$ of $10 \mathrm{mM}$ 2,4-dinitrophenylhydrazine (in $2.5 \mathrm{~N} \mathrm{HCl}$ ) for the sample or $500 \mu \mathrm{L}$ of $2.5 \mathrm{~N} \mathrm{HCl}$ for the blank was added. A dark place was used for storing the samples at room temperature for $1 \mathrm{~h}$, while every $15 \mathrm{~min}$, the samples were vortexed. After $1 \mathrm{~h}$, the samples were centrifuged at $15,000 \times g$ for $5 \mathrm{~min}$ at $4{ }^{\circ} \mathrm{C}$. The supernatant was removed, and $1 \mathrm{~mL}$ of $10 \%$ TCA was added, vortexed, and centrifuged at $15,000 \times \mathrm{g}$ for $5 \mathrm{~min}$ at $4{ }^{\circ} \mathrm{C}$. The supernatant was wasted, and $1 \mathrm{~mL}$ of ethanol-ethyl acetate $(1: 1 \mathrm{v} / \mathrm{v})$ was added, vortexed, and centrifuged at $15,000 \times \mathrm{g}$ for $5 \mathrm{~min}$ at $4{ }^{\circ} \mathrm{C}$. The described washing step was repeated twice. The supernatant was removed, and afterward $1 \mathrm{~mL}$ of $5 \mathrm{M}$ urea (pH 2.3) was added, vortexed, and incubated at $37^{\circ} \mathrm{C}$ for $15 \mathrm{~min}$. The samples were centrifuged at $15,000 \times \mathrm{g}$ for $3 \mathrm{~min}$ at $4{ }^{\circ} \mathrm{C}$, and the absorbance was read at $375 \mathrm{~nm}$. The concentration of protein carbonyls was calculated according to the molar extinction coefficient of dinitrophenylhydrazine.

For the measurement of GSH, $20 \mu \mathrm{L}$ of erythrocyte lysate was added in $5 \%$ TCA and mixed

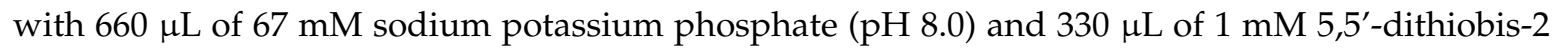
nitrobenzoate. The samples were stored for $45 \mathrm{~min}$ in the dark at room temperature, and afterward the absorbance was read at $412 \mathrm{~nm}$. Creatine kinase was assayed in plasma spectrophotometrically using a kit from Spinreact (Sant Esteve, Spain). 


\subsection{Statistical Analysis}

The Shapiro-Wilk test was used for examining the distribution of all depended parameters, and no significant differences were found compared to normality. Two-way ANOVA (sex (males and females) $\times$ time (pre-exercise and $48 \mathrm{~h}$ after exercise)) with repeated measurements of time were used to analyze isometric torque, ROM, DOMS, CK, protein carbonyls, and GSH. If a significant interaction was obtained, pairwise comparisons were performed through simple main effect analysis. Differences on physical characteristics between males and females were examined by an unpaired Student's $t$-test. The level of statistical significance was set at $\alpha=0.05$, and all the data are presented as mean \pm SD. SPSS version 21.0 was used for all analyses (SPSS Inc., Chicago, IL, USA).

\section{Results}

There were significant differences in physical characteristics between males and females. More specifically, males were taller and heavier compared to females, but had lower body fat $(p<0.05)$. All muscle damage indices did not exhibit any significant effect of group or time-group interaction. Regarding isometric torque, ROM, DOMS, and CK, there was a significant main effect of time $(p<0.001$; Figure 1) since all indices were found to change at $48 \mathrm{~h}$ after exercise for both groups.
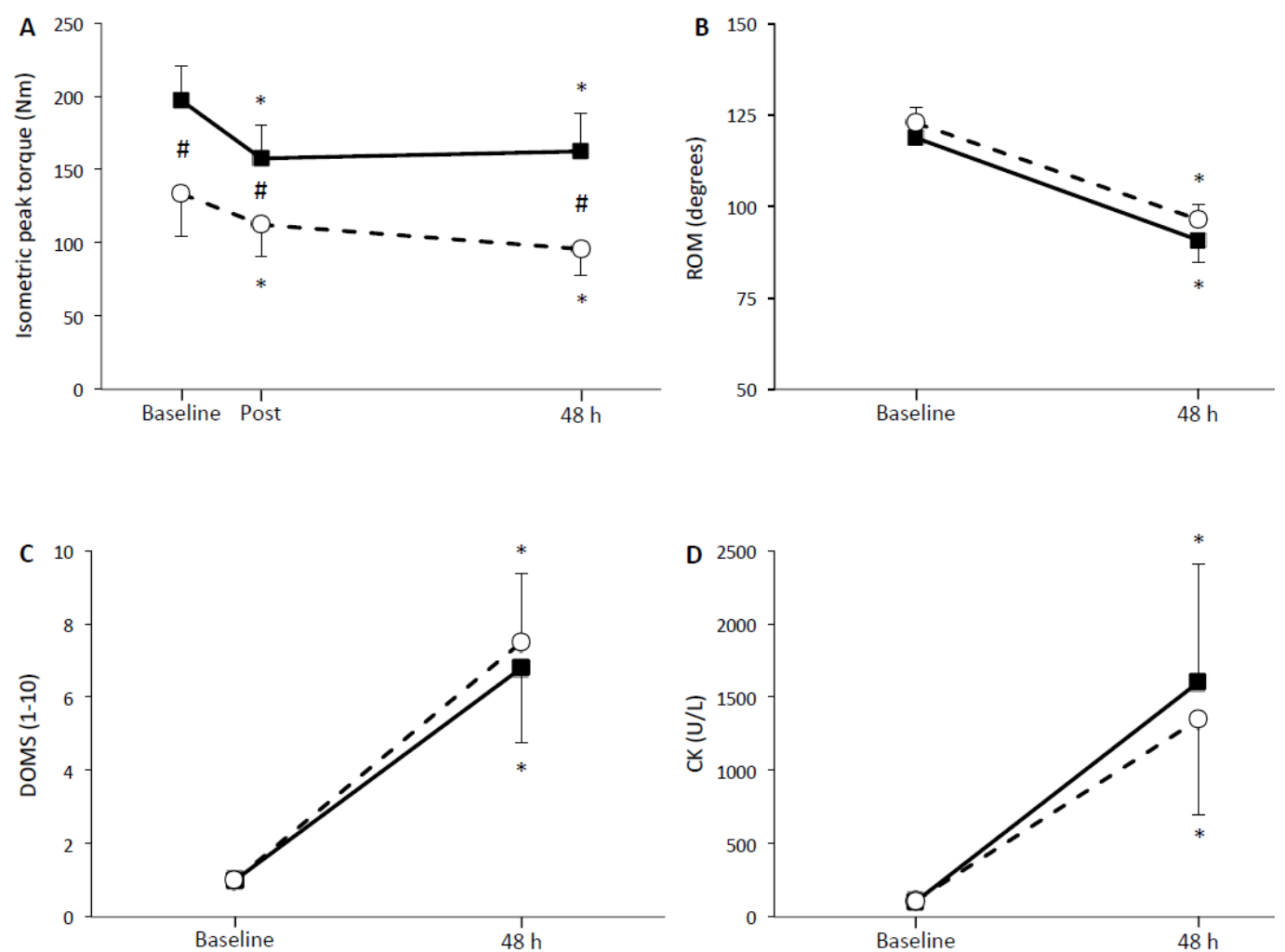

Figure 1. Isometric torque (A) before, immediately after, and $48 \mathrm{~h}$ after eccentric exercise, as well as pain-free range of movement (B), delayed onset muscle soreness (C), and CK (D) before and $48 \mathrm{~h}$ after eccentric exercise for males (solid line) and females (dashed line) (mean $\pm \mathrm{SD}$ ). ${ }^{*}$ Significant difference compared to baseline in the same group. \# Significant difference between the two groups at the same time point.

Similarly, protein carbonyls (Figure 2A) and GSH (Figure 2B) did not reveal any significant main effects of group or time-group interactions. However, there was a significant main effect of time $(p<0.001)$ for the plasma redox status indices, showing significant alterations $48 \mathrm{~h}$ after eccentric exercise compared with baseline values. 

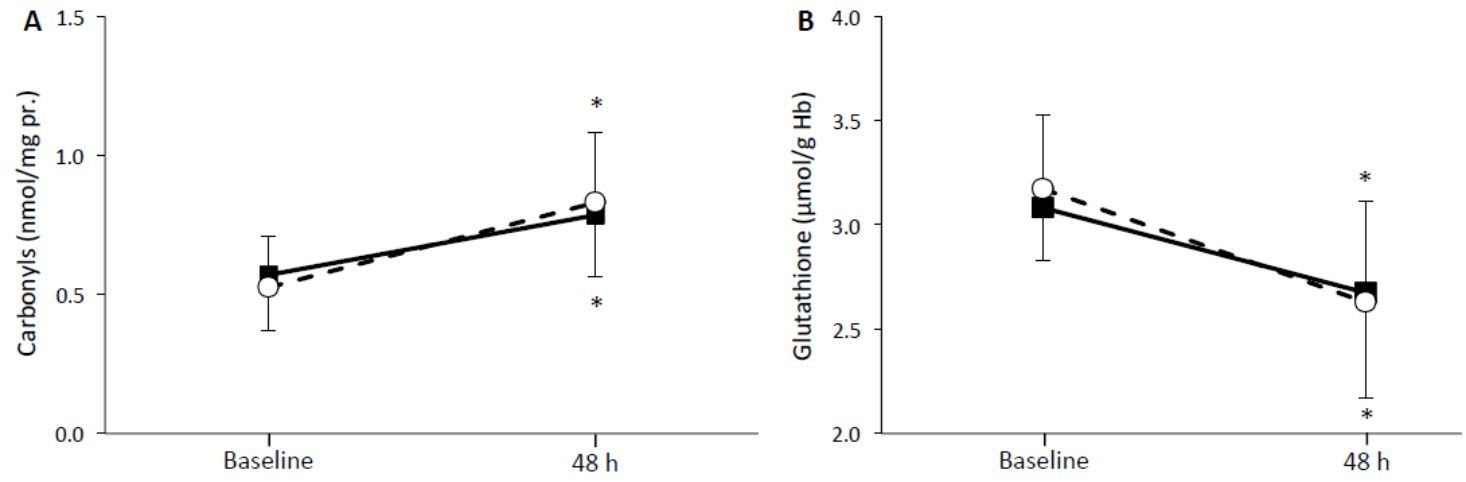

Figure 2. Protein carbonyls (A) and glutathione (B) before and $48 \mathrm{~h}$ after eccentric exercise for males (solid line) and females (dashed line) (mean $\pm \mathrm{SD}$ ). ${ }^{*}$ Significant difference compared to baseline in the same group.

\section{Discussion}

The purpose of the present investigation was to study the possible differences between men and women regarding muscle damage indices and oxidative stress biomarkers in response to eccentric exercise. Despite the fact that eccentric exercise caused muscle damage and altered redox status in both sexes, there were no differences between males and females regarding muscle damage and oxidative stress (in the biomarkers measured) after eccentric exercise.

The present findings are in line with previous literature, where no differences were found between sexes in muscle damage indices after eccentric exercise $[12,14,18]$. In a study of the same nature using muscle biopsies of the vastus lateralis, it was reported that there are no differences in structural damage (z-disk streaming) between sexes in response to unaccustomed eccentric exercise [19]. In the study of Sayers and Clarkson [14], despite the absence of significant differences in muscle damage between men and women, there was a tendency for greater voluntary isometric knee extension torque loss in women compared to men. Similarly, it was reported that the maximal voluntary contraction torque loss was significantly higher in females compared to males immediately post-exercise [20]. However, the same investigators reported that $60 \mathrm{~min}$ after the unaccustomed exercise, no significant difference were observed in torque loss between males and females [20]. It is clear that, when the confounding factor of fatigue was avoided, it can be suggested that a sex difference in torque loss post-exercise induced muscle damage does not exist in the knee extensors.

In previous investigations $[12,13,18]$, the non-significant tendency that was found in women compared to men regarding muscle damage could be explained by the differences in the history of muscle use between sexes. It is known that untrained individuals are more prone to muscle damage, [18] while females, from childhood until young adulthood, seem to be less active and demonstrate more sedentary patterns compared to males [21]. However, in the present investigation, there was no tendency for different responses to eccentric exercise between men and women. The present findings are in line with a previous investigation where a large number of participants clearly showed no differences in DOMS after eccentric exercise of the elbow flexors muscles [13]. However, in the same investigation, a slightly greater loss in ROM for women compared to men was presented [13]. The existence of this tendency was suggested to relay on the possible differences between sexes in the property of connective tissue [22].

In the present investigation, the complete absence of differences regarding the magnitude of muscle damage between men and women could be attributed to the intensity of the exercise, which was maximal voluntary. The absence could also be attributed to the control of female individuals according to their menstrual cycle. It is known that estrogen may play a protective role as an antioxidant as well as a membrane stabilizer during exercise that causes relatively high oxidative stress [23,24]. Moreover, in the present investigation, the eccentric exercise was performed on an isokinetic dynamometer that 
have a number of advantages: (i) during exercise muscle groups are isolated; (ii) muscles perform pure eccentric actions; (iii) eccentric muscle actions can be performed at given intensity (e.g., percent of maximal voluntary contraction) and identical angular velocity between repetitions and bouts.

In the present investigation, it was found that eccentric exercise causes an increase in oxidative stress in the blood, as demonstrated by the similarly changed levels of selected biomarkers. Specifically, protein carbonyls and GSH found to be significant altered at $48 \mathrm{~h}$ after eccentric exercise. Albumin makes up approximately $55 \%$ of total plasma protein content, whereas 10 other abundant proteins account for more than $90 \%$ of all plasma proteins [25]. Therefore, the increased protein carbonyls after exercise should be derived mainly from the oxidation of albumin and the other major plasma proteins. Removal of oxidized proteins from blood is, presumably, a time-consuming process, considering that protein carbonyl concentration remained elevated for a prolonged period $(48 \mathrm{~h})$ after exercise. Certainly, increased production of reactive oxygen and nitrogen species after cessation of exercise also may have contributed to the elevated levels of protein carbonyls after exercise. The GSH data denote that, after exercise, the hepatic GSH supply may not be sufficient to match the enhanced use, resulting in a reduction of blood GSH concentration. There might also be an increase in blood GSH clearance (e.g., increased consumption by muscle) for several days after exercise.

The present observation is in line with previous investigations of our group that eccentric exercise can alter redox status for days after eccentric exercise $[9,10,17]$. However, in the present investigation, no difference was shown in protein carbonyls and GSH $48 \mathrm{~h}$ after eccentric exercise between males and females. It was suggested that mitochondrial oxidative stress is higher in males than females because of the higher levels of estrogens in females upregulating the expression of antioxidants [26]. The control of the menstrual cycle for the females in the present investigation could explain the absence of differences in oxidative stress biomarkers between sexes both in baseline and in response to eccentric exercise. Moreover, the fact that no differences were found between sexes in muscle damage explains the absence of differences also found in oxidative stress. In contrast, in a recent study, it was shown that eccentric exercise caused impaired redox balance only in women [27].

The limited number of individuals used in each group $(n=10)$ could partly explain the lack of differences observed between males and females. In addition, differences could have been observed if other oxidative stress biomarkers had been measured, since different oxidative stress biomarkers exhibit differential time courses during and after exercise [28]. It should be also acknowledged that the findings of the present study are limited to the specific muscle damage and oxidative stress markers employed and for the specific post-exercise time point these markers were measured (i.e., $48 \mathrm{~h}$ ).

\section{Conclusions}

The findings of the present investigation suggest that men and women respond similarly to intense eccentric exercise, which was performed using an isokinetic dynamometer. Additionally, the similar degree of muscle damage induced by eccentric exercise yielded similar redox responses (the biomarkers measured) between sexes.

Author Contributions: Michalis G. Nikolaidis conceived and designed the experiment, performed the experiments, analyzed the data, contributed reagents/materials/analysis tools, and wrote the paper.

Conflicts of Interest: The authors declare no conflict of interest.

\section{References}

1. Hoppeler, H. Exercise-induced ultrastructural changes in skeletal muscle. Int. J. Sports Med. 1986, 7, 187-204. [CrossRef] [PubMed]

2. Paschalis, V.; Nikolaidis, M.G.; Theodorou, A.A.; Panayiotou, G.; Fatouros, I.G.; Koutedakis, Y.; Jamurtas, A.Z. A weekly bout of eccentric exercise is sufficient to induce health-promoting effects. Med. Sci. Sports Exerc. 2011, 43, 64-73. [CrossRef] [PubMed] 
3. Nikolaidis, M.G.; Kyparos, A.; Spanou, C.; Paschalis, V.; Theodorou, A.A.; Panayiotou, G.; Grivas, G.V.; Zafeiridis, A.; Dipla, K.; Vrabas, I.S. Aging is not a barrier to muscle and redox adaptations: Applying the repeated eccentric exercise model. Exp. Gerontol. 2013, 48, 734-743. [CrossRef] [PubMed]

4. Paschalis, V.; Koutedakis, Y.; Jamurtas, A.Z.; Mougios, V.; Baltzopoulos, V. Equal volumes of high and low intensity of eccentric exercise in relation to muscle damage and performance. J. Strength Cond. Res. 2005, 19, 184-188. [PubMed]

5. Armstrong, R.B. Initial events in exercise-induced muscular injury. Med. Sci. Sports Exerc. 1990, 22, 429-435. [PubMed]

6. Paschalis, V.; Nikolaidis, M.G.; Giakas, G.; Jamurtas, A.Z.; Owolabi, E.O.; Koutedakis, Y. Position sense and reaction angle after eccentric exercise: The repeated bout effect. Eur. J. Appl. Physiol. 2008, 103, 9-18. [CrossRef] [PubMed]

7. Radak, Z.; Taylor, A.W.; Ohno, H.; Goto, S. Adaptation to exercise-induced oxidative stress: From muscle to brain. Exerc. Immunol. Rev. 2001, 7, 90-107. [PubMed]

8. Nikolaidis, M.G.; Kyparos, A.; Dipla, K.; Zafeiridis, A.; Sambanis, M.; Grivas, G.V.; Paschalis, V.; Theodorou, A.A.; Papadopoulos, S.; Spanou, C.; et al. Exercise as a model to study redox homeostasis in blood: The effect of protocol and sampling point. Biomarkers 2012, 17, 28-35. [CrossRef] [PubMed]

9. Nikolaidis, M.G.; Paschalis, V.; Giakas, G.; Fatouros, I.G.; Koutedakis, Y.; Kouretas, D.; Jamurtas, A.Z. Decreased blood oxidative stress after repeated muscle-damaging exercise. Med. Sci. Sports Exerc. 2007, 39, 1080-1089. [CrossRef] [PubMed]

10. Paschalis, V.; Nikolaidis, M.G.; Fatouros, I.G.; Giakas, G.; Koutedakis, Y.; Karatzaferi, C.; Kouretas, D.; Jamurtas, A.Z. Uniform and prolonged changes in blood oxidative stress after muscle-damaging exercise. In Vivo 2007, 21, 877-883. [PubMed]

11. Theodorou, A.A.; Nikolaidis, M.G.; Paschalis, V.; Koutsias, S.; Panayiotou, G.; Fatouros, I.G.; Koutedakis, Y.; Jamurtas, A.Z. No effect of antioxidant supplementation on muscle performance and blood redox status adaptations to eccentric training. Am. J. Clin. Nutr. 2011, 93, 1373-1383. [CrossRef] [PubMed]

12. Hicks, K.M.; Onambele, G.L.; Winwood, K.; Morse, C.I. Muscle damage following maximal eccentric knee extensions in males and females. PLoS ONE 2016, 11, e0150848. [CrossRef] [PubMed]

13. Rinard, J.; Clarkson, P.M.; Smith, L.L.; Grossman, M. Response of males and females to high-force eccentric exercise. J. Sports Sci. 2000, 18, 229-236. [CrossRef] [PubMed]

14. Sayers, S.P.; Clarkson, P.M. Force recovery after eccentric exercise in males and females. Eur. J. Appl. Physiol. 2001, 84, 122-126. [CrossRef] [PubMed]

15. Reed, B.G.; Carr, B.R. The Normal Menstrual Cycle and the Control of Ovulation; MDText.com: South Dartmouth, MA, USA, 2000.

16. Minahan, C.; Joyce, S.; Bulmer, A.C.; Cronin, N.; Sabapathy, S. The influence of estradiol on muscle damage and leg strength after intense eccentric exercise. Eur. J. Appl. Physiol. 2015, 115, 1493-1500. [CrossRef] [PubMed]

17. Theodorou, A.A.; Nikolaidis, M.G.; Paschalis, V.; Sakellariou, G.K.; Fatouros, I.G.; Koutedakis, Y.; Jamurtas, A.Z. Comparison between glucose-6-phosphate dehydrogenase-deficient and normal individuals after eccentric exercise. Med. Sci. Sports Exerc. 2010, 42, 1113-1121. [PubMed]

18. Clarkson, P.M.; Nosaka, K.; Braun, B. Muscle function after exercise-induced muscle damage and rapid adaptation. Med. Sci. Sports Exerc. 1992, 24, 512-520. [CrossRef] [PubMed]

19. Stupka, N.; Lowther, S.; Chorneyko, K.; Bourgeois, J.M.; Hogben, C.; Tarnopolsky, M.A. Gender differences in muscle inflammation after eccentric exercise. J. Appl. Physiol. 2000, 89, 2325-2332. [PubMed]

20. Sewright, K.A.; Hubal, M.J.; Kearns, A.; Holbrook, M.T.; Clarkson, P.M. Sex differences in response to maximal eccentric exercise. Med. Sci. Sports Exerc. 2008, 40, 242-251. [CrossRef] [PubMed]

21. Sallis, J.F. Epidemiology of physical activity and fitness in children and adolescents. Crit. Rev. Food Sci. Nutr. 1993, 33, 403-408. [CrossRef] [PubMed]

22. Lahita, R.G. The connective tissue diseases and the overall influence of gender. Int. J. Fertil. Menopausal. Stud. 1996, 41, 156-165. [PubMed]

23. Clarkson, P.M.; Sayers, S.P. Etiology of exercise-induced muscle damage. Can. J. Appl. Physiol. 1999, 24, 234-248. [CrossRef] [PubMed]

24. Tiidus, P.M.; Bombardier, E. Oestrogen attenuates post-exercise myeloperoxidase activity in skeletal muscle of male rats. Acta Physiol. Scand. 1999, 166, 85-90. [CrossRef] [PubMed] 
25. Anderson, N.L.; Anderson, N.G. The human plasma proteome: History, character, and diagnostic prospects. Mol. Cell. Proteomics 2002, 1, 845-867. [CrossRef] [PubMed]

26. Vina, J.; Gambini, J.; Lopez-Grueso, R.; Abdelaziz, K.M.; Jove, M.; Borras, C. Females live longer than males: Role of oxidative stress. Curr. Pharm. Des. 2011, 17, 3959-3965. [CrossRef] [PubMed]

27. Wiecek, M.; Maciejczyk, M.; Szymura, J.; Szygula, Z. Sex differences in oxidative stress after eccentric and concentric exercise. Redox Rep. 2017, 1-8. [CrossRef] [PubMed]

28. Michailidis, Y.; Jamurtas, A.Z.; Nikolaidis, M.G.; Fatouros, I.G.; Koutedakis, Y.; Papassotiriou, I.; Kouretas, D. Sampling time is crucial for measurement of aerobic exercise-induced oxidative stress. Med. Sci. Sports Exerc. 2007, 39, 1107-1113. [CrossRef] [PubMed]

(C) 2017 by the author. Licensee MDPI, Basel, Switzerland. This article is an open access article distributed under the terms and conditions of the Creative Commons Attribution (CC BY) license (http:/ / creativecommons.org/licenses/by/4.0/). 